\title{
Optimal Scheduling of Peer-to-Peer File Dissemination
}

\author{
Jochen Mundinger;, Richard Weber ${ }^{\dagger}$ and Gideon Weiss ${ }^{\ddagger}$
}

\begin{abstract}
Peer-to-peer (P2P) overlay networks such as BitTorrent and Avalanche are increasingly used for disseminating potentially large files from a server to many end users via the Internet. The key idea is to divide the file into many equally-sized parts and then let users download each part (or, for network coding based systems such as Avalanche, linear combinations of the parts) either from the server or from another user who has already downloaded it. However, their performance evaluation has typically been limited to comparing one system relative to another and typically been realized by means of simulation and measurements. In contrast, we provide an analytic performance analysis that is based on a new uplink-sharing version of the well-known broadcasting problem. Assuming equal upload capacities, we show that the minimal time to disseminate the file is the same as for the simultaneous send/receive version of the broadcasting problem. For general upload capacities, we provide a mixed integer linear program (MILP) solution and a complementary fluid limit solution. We thus provide a lower bound which can be used as a performance benchmark for any $\mathrm{P} 2 \mathrm{P}$ file dissemination system. We also investigate the performance of a decentralized strategy, providing evidence that the performance of necessarily decentralized P2P file dissemination systems should be close to this bound and therefore that it is useful in practice.
\end{abstract}

\section{Introduction}

Suppose that $M$ messages of equal length are initially known only at a single source node in a network. The so-called broadcasting problem is about disseminating these $M$ messages to a population of $N$ other nodes in the least possible time, subject to capacity constraints along the links of the network. The assumption is that once a node has received one of the messages it can participate subsequently in sending that message to its neighbouring nodes.

\subsection{P2P file dissemination background and related work}

In recent years, overlay networks have proven a popular way of disseminating potentially large files (such as a new software product or a video) from a single server $S$ to a potentially large group of $N$ end users via the Internet. A number of algorithms and protocols have been suggested, implemented and studied. In particular, much attention has been given to peer-to-peer (P2P) systems such as BitTorrent [9], Slurpie 33, SplitStream [7, Bullet' 20] and Avalanche [12, to name but a few. The key idea is that the file is divided into $M$ parts of equal size and that a given user may download any one of these (or, for network coding

\footnotetext{
*EPFL-IC-LCA, BC203, Station 14, CH-1015 Lausanne, Switzerland, Email: jochen.mundinger@epfl.ch

${ }^{\dagger}$ Statistical Laboratory, Centre for Mathematical Sciences, Wilberforce Road, Cambridge CB3 0WB, UK

${ }^{\ddagger}$ Department of Statistics, University of Haifa, Mount Carmel 31905, Israel
} 
based systems such as Avalanche, linear combinations of these) either from the server or from a peer who has previously downloaded it. That is, the end users collaborate by forming a $\mathrm{P} 2 \mathrm{P}$ network of peers, so they can download from one another as well as from the server. Our motivation for revisiting the broadcasting problem is the performance analysis of such systems.

With the BitTorrent protocol $^{1}$, for example, when the load on the server is heavy, the protocol delegates most of the uploading burden to the users who have already downloaded parts of the file, and who can start uploading those parts to their peers. File parts are typically 1/4 megabyte (MB) in size. An application helps downloading peers to find each other by supplying lists of contact information about randomly selected peers also downloading the file. Peers use this information to connect to a number of neighbours. A full description can be found in 9]. The BitTorrent protocol has been implemented successfully and is deployed widely. A detailed measurement study of the BitTorrent system is reported in 30]. According to [29], BitTorrent's share of the total P2P traffic has reached 53\% in June 2004. For recent measurements of the total P2P traffic on Internet backbones see 19.

Slurpie 33 is a very similar protocol, although, unlike BitTorrent, it does not fix the number of neighbours and it adapts to varying bandwidth conditions. Other P2P overlay networks have also been proposed. For example see SplitStream [7] and Bullet' 20].

More recently, Avalanche ${ }^{2}$ [12, a scheme based on network coding [1] has been suggested. Here, users download linear combinations of file parts rather than individual file parts. This ensures that users do not need to find specific parts in the system, but that any upload by a given user can be of interest to any peer. Thus, network coding can improve performance in a decentralized scenario. Our results apply to any P2P file dissemination system, whether or not it uses network coding.

Performance analysis of $\mathrm{P} 2 \mathrm{P}$ systems for file dissemination has typically been limited to comparing one system relative to another and typically been realized by means of simulation and measurements. We give the makespan, that is the minimal time to fully disseminate the file, of $M$ parts from a server to $N$ end users in a centralized scenario. We thereby provide a lower bound which can be used as a performance benchmark for any P2P file dissemination system. We also investigate the part of the loss in efficiency that is due to the lack of centralized control. Using a theoretical analysis, simulation as well as direct computation, we show that even a naive randomized strategy disseminates the file in an expected time that grows with $N$ in a similar manner to the minimal time achieved with a centralized controller. This suggests that the performance of necessarily decentralized P2P file dissemination systems should still be close to our performance bound so that it is useful in practice.

In this paper, we provide the scheduling background, proofs and discussion of the results in our extended abstracts [27] and [26]. It is essentially Chapter 2 of [23], but we have added Theorem 5 and the part on theoretical bounds in Section [6] In 36] the authors also consider problems concerned with the service capacity of $\mathrm{P} 2 \mathrm{P}$ networks, however, they only give a heuristic argument for the makespan with equal upload capacities when $N$ is of the simple form $2^{n}-1$. In 31] a fluid model for BitTorrent-like networks is introduced and studied, also looking at the effect of incentive mechanisms to address free-riding. Link utilization and fairness are issues in [5]. In [22], also motivated by the BitTorrent protocol and file swarming

\footnotetext{
${ }^{1}$ http://bitconjurer.org/BitTorrent/protocol.html

${ }^{2}$ http://www.research.microsoft.com/ pablo/avalanche.aspx
} 
systems in general, the authors consider a probabilistic model of coupon replication systems. Multi-torrent systems are discussed in [13. There is other related work in [32.

\subsection{Scheduling background and related work}

The broadcasting problem has been considered for different network topologies. Comprehensive surveys can be found in [15] and [16]. On a complete graph, the problem was first solved in [8] and 10. Their communication model was a unidirectional telephone model in which each node can either send or receive one message during each round, but cannot do both. In this model, the minimal number of rounds required is $2 M-1+\left\lfloor\log _{2}(N+1)\right\rfloor$ for even $N$, and $2 M+\left\lfloor\log _{2}(N+1)\right\rfloor-\left\lfloor\frac{M-1+2^{\left\lfloor\log _{2}(N+1)\right\rfloor}}{(N+1) / 2}\right\rfloor$ for odd $N .^{3}$

In [2], the authors considered the bidirectional telephone model in which nodes can both send one message and receive one message simultaneously, but they must be matched pairwise. That is, in each given round, a node can only receive a message from the same node to which it sends a message. They provide an optimal algorithm for odd $N$, which takes $M+\left\lfloor\log _{2} N\right\rfloor$ rounds. For even $N$ their algorithm is optimal up to an additive term of 3 , taking $M+$ $\left\lfloor\log _{2} N\right\rfloor+M / N+2$ rounds.

The simultaneous send/receive model 21] supposes that during each round every user may receive one message and send one message. Unlike the telephone model, it is not required that a user can send a message only to the same user from which it receives a message. The optimal number of rounds turns out to be $M+\left\lfloor\log _{2} N\right\rfloor$ and we will return to this result in Section 3 .

In this paper, we are working with our new uplink-sharing model designed for $\mathrm{P} 2 \mathrm{P}$ file dissemination (cf. Section 21). It is closely related to the simultaneous send/receive model, but is set in continuous time. Moreover, we permit users to have different upload capacities which are the constraints on the data that can be sent per unit of time. This contrasts with previous work in which the aim was to model interactions of processors and so it was natural to assume that all nodes have equal capacities. Our work also differs from previous work in that we are motivated by the evaluation of necessarily decentralized P2P file dissemination algorithms, i.e., ones that can be implemented by the users themselves, rather than by a centralized controller. Our interest in the centralized case is as a basis for comparison and to give a lower bound. We show that in the case of equal upload capacities the optimal number of rounds is $M+\left\lfloor\log _{2} N\right\rfloor$ as for the simultaneous send/receive model. Moreover, we provide two complementary solutions for the case of general upload capacities and investigate the performance of a decentralized strategy.

\subsection{Outlook}

The rest of this paper is organized as follows. In Section 2 we introduce the uplink-sharing model and relate it to the simultaneous send/receive model. Our optimal algorithm for the simultaneous send/receive broadcasting problem is presented in Section 3. We show that it also solves the problem for the uplink-sharing model with equal capacities. In Section 4 we show that the general uplink-sharing model can be solved via a finite number of mixed integer linear programming (MILP) problems. This approach is suitable for a small number of file

\footnotetext{
${ }^{3}$ Bar-Noy, Kipnis and Schieber report a slightly different expression in [2]. This appears to be a transcription error in quoting the result of Cockayne and Thomason.
} 
parts $M$. We provide additional insight through the solution of some special cases. We then consider the limiting case that the file can be divided into infinitely many parts and provide the centralized fluid solution. We extend these results to the even more general situation where different users might have different (disjoint) files of different sizes to disseminate (Section 5 ). This approach is suitable for typical and for large numbers of file parts $M$. Finally, we turn to decentralized algorithms. In Section [6 we evaluate the performance of a very simple and natural randomized strategy, theoretically, by simulation and by direct computation. We provide results in two different information scenarios with equal capacities showing that even this naive algorithm disseminates the file in an expected time whose growth rate with $N$ is similar to the growth rate of the minimal time that we have found for a centralized controller. This suggests that the performance of necessarily decentralized P2P file dissemination systems should still be close to the performance bounds of the previous sections so that they are useful in practice. We conclude and present ideas for further research in Section $\mathbf{7}$

\section{The Uplink-Sharing Model}

We now introduce an abstract model for the file dissemination scenario described in the previous section, focusing on the important features of P2P file dissemination.

Underlying the file dissemination system is the Internet. Thus, each user can connect to every other user and the network topology is a complete graph. The server $S$ has upload capacity $C_{S}$ and the $N$ peers have upload capacities $C_{1}, \ldots, C_{N}$, measured in megabytes per second (MBps). Once a user has received a file part it can participate subsequently in uploading it to its peers (source availability). We suppose that, in principle, any number of users can simultaneously connect to the server or another peer, the available upload capacity being shared equally amongst the open connections (fair sharing). Taking the file size to be $1 \mathrm{MB}$, this means that if $n$ users try simultaneously to download a part of the file (of size $1 / M)$ from the server then it takes $n / M C_{S}$ seconds for these downloads to complete. Observe that the rate at which an upload takes place can both increase and decrease during the time of that upload (varying according to the number of other uploads with which it shares the upload capacity), but we assume that uploads are not interrupted until complete, that is the rate is always positive (continuity). In fact, Lemma 1 below shows that the makespan is not increased if we restrict the server and all peers to carry out only a single upload at a time. We permit a user to download more than one file part simultaneously, but these must be from different sources; only one file part may be transferred from one user to another at the same time. We ignore more complicated interactions and suppose that the upload capacities, $C_{S}, C_{1}, \ldots, C_{N}$, impose the only constraints on the rates at which file parts can be transferred between peers which is a reasonable assumption if the underlying network is not overloaded. Finally, we assume that rates of uploads and downloads do not constrain one another.

Note that we have assumed the download rates to be unconstrained and this might be considered unrealistic. However, we shall show a posteriori in Section 3 that if the upload capacities are equal then additional download capacity constraints do not increase the minimum possible makespan, as long as these download capacities are at least as big. Indeed, this is usually the case in practice.

Typically, $N$ is the order of several thousands and the file size is up to a few gigabytes (GB), so that there are several thousand file parts of size 1/4 MB each. 
Finding the minimal makespan looks potentially very hard as upload times are interdependent and might start at arbitrary points in time. However, the following two observations help simplify it dramatically. As we see in the next section, they also relate the uplink-sharing model to the simultaneous send/receive broadcasting model.

Lemma 1 In the uplink-sharing model the minimal makespan is not increased by restricting attention to schedules in which the server and each of the peers only carry out a single upload at a time.

Proof. Identify the server as peer 0 and, for each $i=0,1, \ldots, N$ consider the schedule of peer $i$. We shall use the term job to mean the uploading of a particular file part to a particular peer. Consider the set of jobs, say $J$, whose processing involves some sharing of the upload capacity $C_{i}$. Pick any job, say $j$, in $J$ which is last in $J$ to finish and call the time at which it finishes $t_{f}$. Now fair sharing and continuity imply that job $j$ is amongst the last to start amongst all the jobs finishing before or at time $t_{f}$. To see this, note that if some job $k$ were to start later than $j$, then (by fair sharing and continuity) $k$ must receive less processing than job $j$ by time $t_{f}$ and so cannot have finished by time $t_{f}$. Let $t_{s}$ denote the starting time of job $j$.

We now modify the schedule between time $t_{s}$ and $t_{f}$ as follows. Let $K$ be the set of jobs with which job $j$ 's processing has involved some sharing of the upload capacity. Let us re-schedule job $j$ so that it is processed on its own between times $t_{f}-1 / C_{i} M$ and $t_{f}$. This consumes some amount of upload capacity that had been devoted to jobs in $K$ between $t_{f}-1 / C_{i} M$ and $t_{f}$. However, it releases an exactly equal amount of upload capacity between times $t_{s}$ and $t_{f}-1 / C_{i} M$ which had been used by job $j$. This can now be allocated (using fair sharing) to processing jobs in $K$.

The result is that $j$ can be removed from the set $J$. All jobs finish no later than they did under the original schedule. Moreover, job $j$ starts later than it did under the original schedule and the scheduling before time $t_{s}$ and after time $t_{f}$ is not affected. Thus, all jobs start no earlier than they did under the original schedule. This ensures that the source availability constraints are satisfied and that we can consider the upload schedules independently. We repeatedly apply this argument until set $J$ is empty.

Using Lemma 1, a similar argument shows the following result.

Lemma 2 In the uplink-sharing model the minimal makespan is not increased by restricting attention to schedules in which uploads start only at times that other uploads finish or at time 0 .

Proof. By the previous Lemma it suffices to consider schedules in which the server and each of the peers only carry out a single upload at a time. Consider the joint schedule of all peers $i=0,1, \ldots, N$ and let $J$ be the set of jobs that start at a time other than 0 at which no other upload finishes. Pick a job, say $j$, that is amongst the first in $J$ to start, say at time $t_{s}$. Consider the greatest time $t_{f}$ such that $t_{f}<t_{s}$ and $t_{f}$ is either 0 or the time that some other upload finishes and modify the schedule so that job $j$ already starts at time $t_{f}$.

The source availability constraints are still satisfied and all uploads finish no later than they did under the original schedule. Job $j$ can be removed from the set $J$ and the number of jobs in $J$ that start at time $t_{s}$ is decreased by 1 , although there might now be more (but at most $N$ in total) jobs in $J$ that start at the time that job $j$ finished in the original schedule. 
But this time is later than $t_{s}$. Thus, we repeatedly apply this argument until the number of jobs in $J$ that start at time $t_{s}$ becomes 0 and then move along to jobs in $J$ that are now amongst the first in $j$ to start at time $t_{s}^{\prime}>t_{s}$. Note that once a job has been removed from $J$, it will never be included again. Thus we continue until the set $J$ is empty.

\section{Centralized Solution for Equal Capacities}

In this section, we give the optimal centralized solution of the uplink-sharing model of the previous section with equal upload capacities. We first consider the simultaneous send/receive broadcasting model in which the server and all users have upload capacity of 1 . The following theorem provides a formula for the minimal makespan and a centralized algorithm that achieves it is contained in the proof.

This agrees with a result of Bar-Noy, Kipnis and Schieber [2], who obtained it as a byproduct of their result on the bidirectional telephone model. However, they required pairwise matchings in order to apply the results from the telephone model. So, for the simultaneous send/receive model, too, they use perfect matching in each round for odd $N$, and perfect matching on $N-2$ nodes for even $N$. As a result, their algorithm differs for odd and even $N$ and it is substantially more complicated, to describe, implement and prove to be correct, than the one we present within the proof of Theorem 1 Theorem 1 has been obtained also by Kwon and Chwa 21, via an algorithm for broadcasting in hypercubes. By contrast, our explicitly constructive proof makes the structure of the algorithm very easy to see. Moreover, it makes the proof of Theorem 3, that is, the result for the uplink-sharing model, a trivial consequence (using Lemmata 1 and 2).

Essentially, the $\log _{2} N$-scaling is due to the P2P approach. This compares favourably to the linear scaling of $N$ that we would obtain for a fixed set of servers. The factor of $1 / M$ is due to splitting the file into parts.

Theorem 1 In the simultaneous send/receive model with all upload and download capacities equal to 1 , the minimum number of rounds is $M+\left\lfloor\log _{2} N\right\rfloor$, each round taking up $1 / M$ units of time. Equivalently, for all $M, N$, the minimal makespan is

$$
T^{*}=1+\frac{\left\lfloor\log _{2} N\right\rfloor}{M} .
$$

Proof. Suppose that $N=2^{n}-1+x$, for $x=1, \ldots, 2^{n}$. So $n=\left\lfloor\log _{2} N\right\rfloor$. The fact that $M+n$ is a lower bound on the number of rounds is straightforwardly seen as follows. There are $M$ different file parts and the server can only upload one file part (or one linear combination of file parts) in each round. Thus, it takes at least $M$ rounds until the server has made sufficiently many uploads of file parts (or linear combinations of file parts) that the whole file can be recovered. The last of these $M$ uploads by the server contains information that is essential to recovering the file, but this information is now known to only the server and one peer. It must takes at least $n$ further rounds to disseminate this information to the other $N-1$ peers.

Now we show how the bound can be achieved. The result is trivial for $M=1$. It is instructive to consider the case $M=2$ explicitly. If $n=0$ then $N=1$ and the result is 
trivial. If $n=1$ then $N$ is 2 or 3 . Suppose $N=3$. In the following diagram each line corresponds to a round; each column to a peer. The entries denote the file part that the peer downloads that round. The bold entries indicate downloads from the server; un-bold entries indicate downloads from a peer who has the corresponding part.

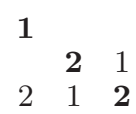

Thus, dissemination of the two file parts to the 3 users can be completed in 3 rounds. The case $N=2$ is even easier.

If $n \geq 2$, then in rounds 2 to $n$ each user uploads his part to a peer who has no file part and the server uploads part 2 to a peer who has no file part. We reach a point, shown below, at which a set of $2^{n-1}$ peers have file part 1 , a set of $2^{n-1}-1$ peers have file part 2 , and a set of $x$ peers have no file part (those denoted by $* \cdots *$ ). Let us call these three sets $A_{1}, A_{2}$ and $A_{0}$, respectively.

1

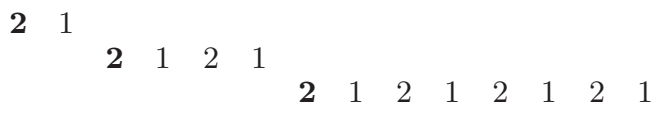

In round $n+1$ we let peers in $A_{1}$ upload part 1 to $2^{n-1}-\lfloor x / 2\rfloor$ peers in $A_{2}$ and to $\lfloor x / 2\rfloor$ peers in $A_{0}$ (If $x=1$, to $2^{n-1}-1$ peers in $A_{2}$ and to 1 peer in $A_{0}$ ). Peers in $A_{2}$ upload part 2 to $2^{n-1}-\lceil x / 2\rceil$ peers in $A_{1}$ and to another $\lceil x / 2\rceil-1$ peers in $A_{0}$. The server uploads part 2 to a member of $A_{0}$ (If $x=1$, to a member of $A_{1}$ ). Thus, at the end of this round $2^{n}-x$ peers have both file parts, $x$ peers have only file part 1 , and $x-1$ peers have only file part 2 . One more round (round $n+2$ ) is clearly sufficient to complete the dissemination.

Now consider $M \geq 3$. The server uploads part 1 to one peer in round 1 . In rounds $j=2, \ldots, \min \{n, M-1\}$, each peer who has a file part uploads his part to another peer who has no file part and the server uploads part $j$ to a peer who has no file part. If $M \leq n$, then in rounds $M$ to $n$ each peer uploads his part to a peer who has no file part and the server uploads part $M$ to a peer who has no file part. As above, we illustrate this with a diagram. Here we show the first $n$ rounds in the case $M \leq n$.

1

21

$\begin{array}{llll}3 & 1 & 2 & 1\end{array}$

$\begin{array}{llllllll}4 & 1 & 2 & 1 & 3 & 1 & 2 & 1\end{array}$

$\begin{array}{lllll}\mathbf{M} & 1 & \cdots & 2 & 1\end{array}$

$\begin{array}{llllll} & \mathbf{M} & 1 & \cdots & 2 & 1\end{array} * \cdots *$

When round $n$ ends, $2^{n}-1$ peers have one file part and $x$ peers have no file part. The number of peers having file part $i$ is given in the second column of Table 1. In this table any entry which evaluates to less than 1 is to be read as 0 (so, for example, the bottom two entries in 


\begin{tabular}{cllllll}
\hline Part & \multicolumn{5}{c}{ Numbers of the file parts at the ends of rounds } \\
& $n$ & $n+1$ & $n+2$ & $n+3$ & $\cdots$ & $n+M-1$ \\
\hline 1 & $2^{n-1}$ & $2^{n}$ & $N$ & $N$ & $\cdots$ & $N$ \\
2 & $2^{n-2}$ & $2^{n-1}$ & $2^{n}$ & $N$ & $\cdots$ & $N$ \\
3 & $2^{n-3}$ & $2^{n-2}$ & $2^{n-1}$ & $2^{n}$ & $\cdots$ & $N$ \\
4 & $2^{n-4}$ & $2^{n-3}$ & $2^{n-2}$ & $2^{n-1}$ & $\cdots$ & $N$ \\
$\vdots$ & $\vdots$ & $\vdots$ & $\vdots$ & $\vdots$ & & $\vdots$ \\
$M-2$ & $2^{n-M+2}$ & $2^{n-M+3}$ & $2^{n-M+4}$ & $2^{n-M+5}$ & $\cdots$ & $N$ \\
$M-1$ & $2^{n-M+1}$ & $2^{n-M+2}$ & $2^{n-M+3}$ & $2^{n-M+4}$ & $\cdots$ & $2^{n}$ \\
$M$ & $2^{n-M+1}-1$ & $2^{n-M+2}-1$ & $2^{n-M+3}-1$ & $2^{n-M+4}-1$ & $\cdots$ & $2^{n}-1$ \\
\hline
\end{tabular}

Table 1: Number of file part replica as obtained with our algorithm.

\begin{tabular}{lll}
\hline set & peers in the set have & number of peers in set \\
\hline$B_{12}$ & parts 1 and 2 & $2^{n-1}-\lfloor x / 2\rfloor$ \\
$B_{1 p}$ & part 1 and a part other than 1 or 2 & $2^{n-1}-\lceil x / 2\rceil$ \\
$B_{1}$ & just part 1 & $x$ \\
$B_{2}$ & just part 2 & $\lfloor x / 2\rfloor$ \\
$B_{p}$ & just a part other than 1 or 2 & $\lceil x / 2\rceil-1$ \\
\hline
\end{tabular}

Table 2: File parts held by various sets of peers at the end of round $n+1$.

column 2 and the bottom entry in column 3 are 0 for $n=M-2$ ). Now in round $n+1$, by downloading from every peer who has a file part, and downloading part $\min \{n+1, M\}$ from the server, we can obtain the numbers shown in the third column. Moreover, we can easily arrange so that peers can be divided into the sets $B_{12}, B_{1 p}, B_{1}, B_{2}$ and $B_{p}$ as shown in Table 2 In round $n+2, x-1$ of the peers in $B_{1}$ upload part 1 to peers in $B_{2}$ and $B_{p}$. Peers in $B_{12}$ and $B_{2}$ each upload part 2 to the peers in $B_{1 p}$ and to $\lceil x / 2\rceil$ of the peers in $B_{1}$. The server and the peers in $B_{1 p}$ and $B_{p}$ each upload a part other than 1 or 2 to the peers in $B_{12}$ and to the other $\lfloor x / 2\rfloor$ peers in $B_{1}$. The server uploads part $\min \{n+2, M\}$ and so we obtain the numbers in the fourth column of Table 1 Now all peers have part 1 and so it can be disregarded subsequently. Moreover, we can make the downloads from the server, $B_{1 p}$ and $B_{p}$ so that (disregarding part 1 ) the number of peers who ultimately have only part 3 is $\lfloor x / 2\rfloor$. This is possible because the size of $B_{p}$ is no more than $\lfloor x / 2\rfloor$; so if $j$ peers in $B_{p}$ have part 3 then we can upload part 3 to exactly $\lfloor x / 2\rfloor-j$ peers in $B_{1}$. Thus, a similar partitioning into sets as in Table 2 will hold as we start step $n+3$ (when parts 2 and 3 takes over the roles of parts 1 and 2 respectively).

We continue similarly in subsequent rounds, until at the end of round $n+M-1$, all peers have parts $1, \ldots, M-2,2^{n}-x$ peers also have both part $M-1$ and part $M, x$ peers also have only part $M-1$, and $x-1$ peers also have only part $M$. It now takes just a final round to ensure that all peers have parts $M-1$ and $M$.

Note that the optimal strategy above follows two principles. As many different peers as possible obtain file parts early on so that they can start uploading themselves and the maximal possible upload capacity is used. Moreover, there is a certain balance in the upload of different file parts so that no part gets circulated too late. 
It is interesting that not all the available upload capacity is used. Suppose $M \geq 2$. Observe that in round $k$, for each $k=n+2, \ldots, n+M-1$, only $x-1$ of the $x$ peers (in set $B_{1}$ ) who have only file part $k-n-1$ make an upload. This happens $M-2$ times. Also, in round $n+M$ there are only $2 x-1$ uploads, whereas $N+1$ are possible. Overall, we use $N+M-2 x$ less uploads than we might. It can be checked that this number is the same for $M=1$.

Suppose we were to follow a schedule that uses only $x$ uploads during round $n+1$, when the last peer gets its first file part. We would be using $2^{n}-x$ less uploads than we might in this round. Since $2^{n}-x \leq N+M-2 x$, we see that the schedule used in the proof above wastes at least as many uploads. So the mathematically interesting question arises as to whether or not it is necessary to use more than $x$ uploads in round $n+1$. In fact, $(N+M-2 x)-\left(2^{n}-x\right)=M-1$, so, in terms of the total number of uploads, such a scheduling could still afford not to use one upload during each of the last $M-1$ rounds. The question is whether or not each file part can be made available sufficiently often.

The following example shows that if we are not to use more than $x$ uploads in round $n+1$ we will have to do something quite subtle. We cannot simply pick any $x$ out of the $2^{n}$ uploads possible and still hope that an optimal schedule will be shiftable: by which we mean that the number of copies of part $j$ at the end of round $k$ will be the same as the number of copies of part $j-1$ at the end of round $k-1$. It is the fact that the optimal schedule used in Theorem 1 is shiftable that makes its optimality so easy to see.

Example 1 Suppose $M=4$ and $N=13=2^{3}+6-1$, so $M+\left\lfloor\log _{2} N\right\rfloor=7$. If we follow the same schedule as in Theorem 1 , we reach after round 3 ,

\section{$\begin{array}{lllllll}1 & 2 & 1 & 3 & 1 & 2 & 1\end{array}$}

Now if we only make $x=6$ uploads during round 4, then there are eight ways to choose which six parts to upload and which two parts not to upload. One can check that in no case is it possible to arrange so that once this is done and uploads are made for round 5 then the resulting state has the same numbers of parts 2, 3 and 4, respectively, as the numbers of parts 1, 2 and 3 at the end of round 4. That is, there is no shiftable optimal schedule. In fact, if our six uploads has been four part $1 s$ and two part $2 s$, then it would not even be possible to achieve (11).

In some cases, we can achieve (1), if we relax the demand that the schedule be shiftable. Indeed, we conjecture that this is always possible for at least one schedule that uses only $x$ uploads during round $n+1$. However, the fact that we cannot use essentially the same strategy in each round makes the general description of a non-shiftable optimal schedule very complicated. Our aim has been to find an optimal (shiftable) schedule that is easy to describe. We have shown that this is possible if we do use the spare capacity at round $n+1$. For practical purposes this is desirable anyway, since even if it does not affect the makespan it is better if users obtain file parts earlier.

When $x=2^{n}$ our schedule can be realized using matchings between the $2^{n}$ peers holding the part that is to be completed next and the server together with the $2^{n}-1$ peers holding the remaining parts. But otherwise this is not always possible to schedule only with matchings. This is why our solution would not work for the more constrained telephone-like model considered in [2] (where, in fact, the answer differs as $N$ is even or odd). to describe. 
The solution of the simultaneous send/receive broadcasting model problem now gives the solution of our original uplink-sharing model when all capacities are the same.

Theorem 2 Consider the uplink-sharing model with all upload capacities equal to 1. The minimal makespan is given by (11), for all $M, N$, the same as in the simultaneous send/receive model with all upload capacities equal to 1.

Proof. Note that under the assumptions of the theorem and with application of Lemmas 1 and2, the optimal solution to the uplink-sharing model is the same as that of the simultaneous send/receive broadcast model when all upload capacities equal to 1.

In the proof of Theorem 1 we explicitly gave an optimal schedule which also satisfies the constraints that no peer downloads more than a single file part at a time. Thus, we also have the following result.

Theorem 3 In the uplink-sharing model with all upload capacities equal to 1, constraining the peers' download rates to 1 does not further increase the minimal makespan.

\section{Centralized Solution for General Capacities}

We now consider the optimal centralized solution in the general case of the uplink-sharing model in which the upload capacities may be different. Essentially, we have an unusual type of precedence-constrained job scheduling problem. In Section 4.1] we formulate it as a mixed integer linear program (MILP). The MILP can also be used to find approximate solutions of bounded size of sub-optimality. In practice, it is suitable for a small number of file parts $M$. We discuss its implementation in Section 4.2. Finally, we provide additional insight into the solution with different capacities by considering special choices for $N$ and $M$ when $C_{1}=C_{2}=\cdots=C_{N}$, but $C_{S}$ might be different (Sections 4.3 and 4.4).

\subsection{MILP formulation}

In order to give the MILP formulation, we need the following Lemma. Essentially, it shows that time can be discretized suitably.

Lemma 3 Consider the uplink-sharing model and suppose all uplink capacities are integer multiples of a common time unit. Then there exists $\tau$, such that under an optimal schedule all uploads start and finish at integer multiples of $\tau$.

Proof. Rescale time so that $C_{S}, C_{1}, \ldots, C_{N}$ are all integers and let $L$ be their least common multiple. The time that the first job completes must be an integer multiple of $1 / M L$. All remaining jobs are of sizes $1 / M$ or $1 / M-\left(1 / M C_{j}\right) C_{i}$ for various $C_{i} \leq C_{j}$. These are also integer multiples of $1 / M L$. Repeating this, we find that the time that the second job completes, and the lengths of all remaining jobs at this point must be integer multiples of $1 /(M L)^{2}$. Repeating further, we find that $\tau=1 /(M L)^{M N}$ suffices.

We next show how the solution to the general problem can be found by solving a number of linear programs. Let time interval $t$ be the interval $[t \tau, t \tau+\tau), t=0, \ldots$. Identify the server as peer 0 . Let $x_{i j k}(t)$ be 1 or 0 as peer $i$ downloads file part $k$ from peer $j$ during interval $t$ or not. Let $p_{i k}(t)$ denote the proportion of file part $k$ that peer $i$ has downloaded 
by time $t$. Our problem is then is to find the minimal $T$ such that the optimal value of the following MILP is $M N$. Since this $T$ is certainly greater than $1 / C_{S}$ and less than $N / C_{S}$, we can search for its value by a simple bisection search, solving this LP for various $T$ :

$$
\operatorname{maximize} \sum_{i, k} p_{i k}(T)
$$

subject to the constraints given below. The source availability constraint (6) guarantees that a user has completely downloaded a part before he can upload it to his peers. The connection constraint (7) requires that each user only carries out a single upload at a time. This is justified by Lemma 1 which also saves us another essential constraint and variable to control the actual download rates: The single user downloading from peer $j$ at time $t$ will do so at rate $C_{j}$ as expressed in the link constraint (5). Continuity and stopping constraints (8, 9) require that a download that has started will not be interrupted until completion and then be stopped. The exclusivity constraint (10) ensures that each user downloads a given file part only from one peer, not from several ones. Stopping and exclusivity constraints are not based on assumptions, but obvious constraints to exclude redundant uploads.

\section{Regional constraints}

$$
\begin{gathered}
x_{i j k}(t) \in\{0,1\} \text { for all } i, j, k, t \\
p_{i k}(t) \in[0,1] \text { for all } i, k, t
\end{gathered}
$$

\section{Link constraints between variables}

$$
p_{i k}(t)=M \tau \sum_{t^{\prime}=0}^{t-\tau} \sum_{j=0}^{N} x_{i j k}\left(t^{\prime}\right) C_{j} \text { for all } i, k, t
$$

\section{Essential constraints}

$$
\begin{gathered}
x_{i j k}(t)-\xi_{j k}(t) \leq 0 \text { for all } i, j, k, t \quad \text { (Source availability constraint) } \\
\sum_{i, k} x_{i j k}(t) \leq 1 \text { for all } j, t \quad \text { (Connection constraint) } \\
x_{i j k}(t)-\xi_{i k}(t+1)-x_{i j k}(t+1) \leq 0 \text { for all } i, j, k, t \quad \text { (Continuity constraint) } \\
x_{i j k}(t)+\xi_{i k}(t) \leq 1 \text { for all } i, j, k, t \quad \text { (Stopping constraint) } \\
\sum_{j} x_{i j k}(t) \leq 1 \text { for all } i, k, t \quad \text { (Exclusivity constraint) }
\end{gathered}
$$

\section{Initial conditions}

$$
\begin{gathered}
p_{0 k}(0)=1 \text { for all } k \\
p_{i k}(0)=0 \text { for all } i, k
\end{gathered}
$$

Constraints (81)-(6]) have been linearized. Background can be found in [34. For this, we used the auxiliary variable $\xi_{i k}(t)=\mathbf{1}\left\{p_{i k}(t)=1\right\}$. This definition can be expressed through the following linear constraints.

\section{Linearization constraints}

$$
\begin{gathered}
\xi_{i k}(t) \in\{0,1\} \text { for all } i, k, t \\
p_{i k}(t)-\xi_{i k}(t) \geq 0 \text { and } p_{i k}(t)-\xi_{i k}(t)<1 \text { for all } i, k, t
\end{gathered}
$$


It can be checked that together with (8)-(6), indeed, this gives

$$
\begin{gathered}
x_{i j k}(t)=1 \text { and } p_{i k}(t+1)<1 \Longrightarrow x_{i j k}(t+1)=1 \text { for all } i, j, k, t \\
p_{i k}(t)=1 \Longrightarrow x_{i j k}(t)=0 \text { for all } i, j, k, t \\
p_{j k}(t)<1 \Longrightarrow x_{i j k}(t)=0 \text { for all } i, j, k, t
\end{gathered}
$$

that is, continuity, stopping and source availability constraints respectively.

\subsection{Implementation of the MILP}

MILPs are well-understood and there exist efficient computational methods and program codes. The simplex method introduced by Dantzig in 1947, in particular, has been found to yield an efficient algorithm in practice as well as providing insight into the theory. Since then, the method has been specialized to take advantage of the particular structure of certain classes of problems and various interior point methods have been introduced. For integer programming there are branch-and-bound, cutting plane (branch-and-cut) and column generation (branch-and-price) methods as well as dynamic programming algorithms. Moreover, there are various approximation algorithms and heuristics. These methods have been implemented in many commercial optimization libraries such as OSL or CPLEX. For further reading on these issues the reader is referred to [28, [4] and [38].

Thus, implementing and solving the MILPs gives the minimal makespan solution. Although, as the numbers of variables and constraints in the LP grows exponentially in $N$ and $M$, this approach is not practical for large $N$ and $M$.

Even so, we can use the LP formulation to obtain a bounded approximation to the solution. If we look at the problem with a greater $\tau$, then the job end and start times are not guaranteed to lie at integer multiples of $\tau$. However, if we imagine that each job does take until the end of an $\tau$-length interval to finish (rather than finishing before the end), then we will overestimate the time that each job takes by at most $\tau$. Since there are $N M$ jobs in total, we overestimate the total time taken by at most $N M \tau$. Thus, the approximation gives us an upper bound on the time taken and is at most $N M \tau$ greater than the true optimum. So we obtain both upper and lower bounds on the minimal makespan. Even for this approximation, the computing required is formidable for large $N$ and $M$.

\subsection{Insight for special cases with small $N$ and $M$}

We now provide some insight into the minimal makespan solution with different capacities by considering special choices for $N$ and $M$ when $C_{1}=C_{2}=\cdots=C_{N}$, but $C_{S}$ might be different. This addresses the case of the server having a significantly higher upload capacity than the end users.

Suppose $N=2$ and $M=1$, that is, the file has not been split. Only the server has the file initially, thus either (a) both peers download from the server, in which case the makespan is $T=2 / C_{S}$, or (b) one peer downloads from the server and then the second peer downloads from the first; in this case $T=1 / C_{S}+1 / C_{1}$. Thus, the minimal makespan is $T^{*}=1 / C_{S}+\min \left\{1 / C_{S}, 1 / C_{1}\right\}$.

If $N=M=2$ we can again adopt a brute force approach. There are 16 possible cases, each specifying the download source that each peer uses for each part. These can be reduced to four by symmetry. 
Case A: Everything is downloaded from the server. This is effectively the same as case (a) above. When $C_{1}$ is small compared to $C_{S}$, this is the optimal strategy.

Case B: One peer downloads everything from the server. The second peer downloads from the first. This is as case (b) above, but since the file is split in two, $T$ is less.

Case C: One peer downloads from the server. The other peer downloads one part of the file from the server and the other part from the first peer.

Case D: Each peer downloads exactly one part from the server and the other part from the other peer. When $C_{1}$ is large compared to $C_{S}$, this is the optimal strategy.

In each case, we can find the optimal scheduling and hence the minimal makespan. This is shown in Table 3

\begin{tabular}{cl}
\hline case & makespan \\
\hline $\mathrm{A}$ & $\frac{2}{C_{S}}$ \\
$\mathrm{~B}$ & $\frac{1}{2 C_{S}}+\frac{1}{2 C_{1}}+\max \left(\frac{1}{2 C_{S}}, \frac{1}{2 C_{1}}\right)$ \\
$\mathrm{C}$ & $\frac{1}{2 C_{S}}+\max \left(\frac{1}{C_{S}}, \frac{1}{2 C_{1}}\right)$ \\
$\mathrm{D}$ & $\frac{1}{C_{S}}+\frac{1}{2 C_{1}}$ \\
\hline
\end{tabular}

Table 3: Minimal makespan in the four possible cases when $N=M=2$.

The optimal strategy arises from $\mathrm{A}, \mathrm{C}$ or $\mathrm{D}$ as $C_{1} / C_{S}$ lies in the intervals $[0,1 / 3],[1 / 3,1]$ or $[1, \infty)$ respectively. In $[1, \infty), \mathrm{B}$ and D yield the same. See Figure 1 Note that under the optimal schedule for case $\mathrm{C}$ one peer has to wait while the other starts downloading. This illustrates that greedy-type distributed algorithms may not be optimal and that restricting uploaders to a single upload is sometimes necessary for an optimal scheduling (cf. Section 2).

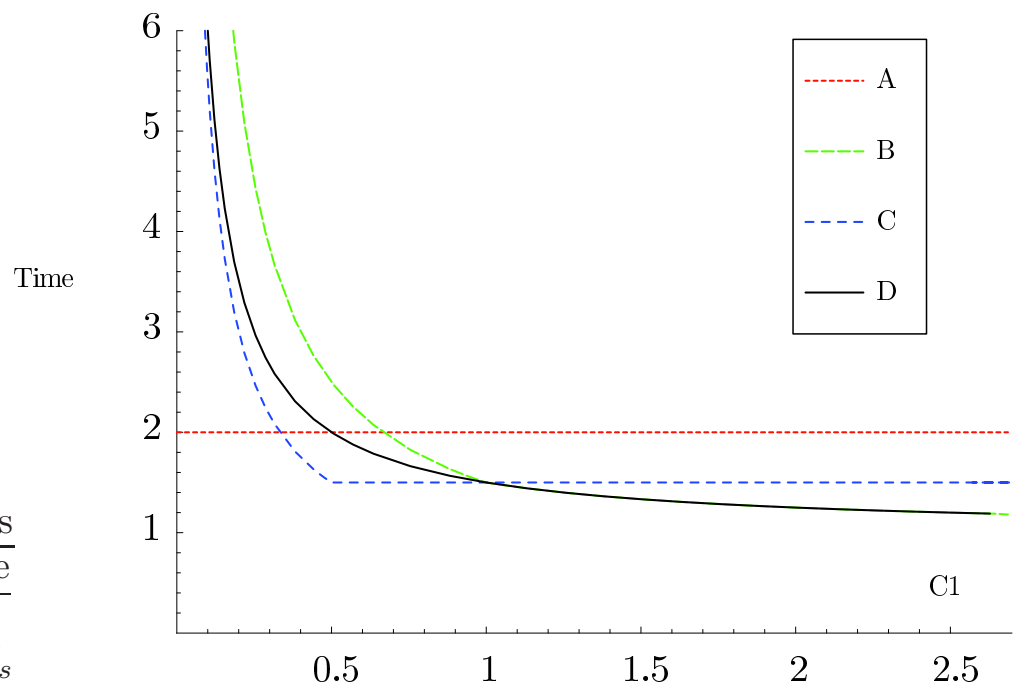

Figure 1: Minimal makespan as a function of $C_{1} / C_{S}$ in the four possible cases when $N=M=2$. 


\subsection{Insight for special cases with large $M$}

We still assume $C_{1}=C_{2}=\cdots=C_{N}$, but $C_{S}$ might be different. In the limiting case that the file can be divided into infinitely many parts, the problem can be easily solved for any number $N$ of users. Let each user download a fraction $1-\alpha$ directly from the server at rate $C_{S} / N$ and a fraction $\alpha /(N-1)$ from each of the other $N-1$ peers, at rate $\min \left\{C_{S} / N, C_{1} /(N-1)\right\}$ from each. The makespan is minimized by choosing $\alpha$ such that the times for these two downloads are equal, if possible. Equating them, we find the minimal makespan as follows.

Case 1: $C_{1} /(N-1) \leq C_{S} / N$ :

$$
\frac{(1-\alpha) N}{C_{S}}=\frac{\alpha}{C_{1}} \quad \Longrightarrow \quad \alpha=\frac{N C_{1}}{C_{S}+N C_{1}} \quad \Longrightarrow \quad T=\frac{N}{C_{S}+N C_{1}}
$$

Case 2: $C_{1} /(N-1) \geq C_{S} / N$ :

$$
\frac{(1-\alpha) N}{C_{S}}=\frac{\alpha N}{(N-1) C_{S}} \quad \Longrightarrow \quad \alpha=\frac{N-1}{N} \quad \Longrightarrow \quad T=\frac{1}{C_{S}} .
$$

In total, there are $N \mathrm{MB}$ to upload and the total available upload capacity is $C_{S}+N C_{1}$ MBps. Thus, a lower bound on the makespan is $N /\left(C_{S}+N C_{1}\right)$ seconds. Moreover, the server has to upload his file to at least one user. Hence another lower bound on the makespan is $1 / C_{S}$. The former bound dominates in case 1 and we have shown that it can be achieved. The latter bound dominates in case 2 and we have shown that it can be achieved. As a result, the minimal makespan is

$$
T^{*}=\max \left\{\frac{1}{C_{S}}, \frac{N}{C_{S}+N C_{1}}\right\} .
$$

Figure 2 shows the minimal makespan when the file is split in 1,2 and infinitely many file parts when $N=2$. It illustrates how the makespan decreases with $M$.

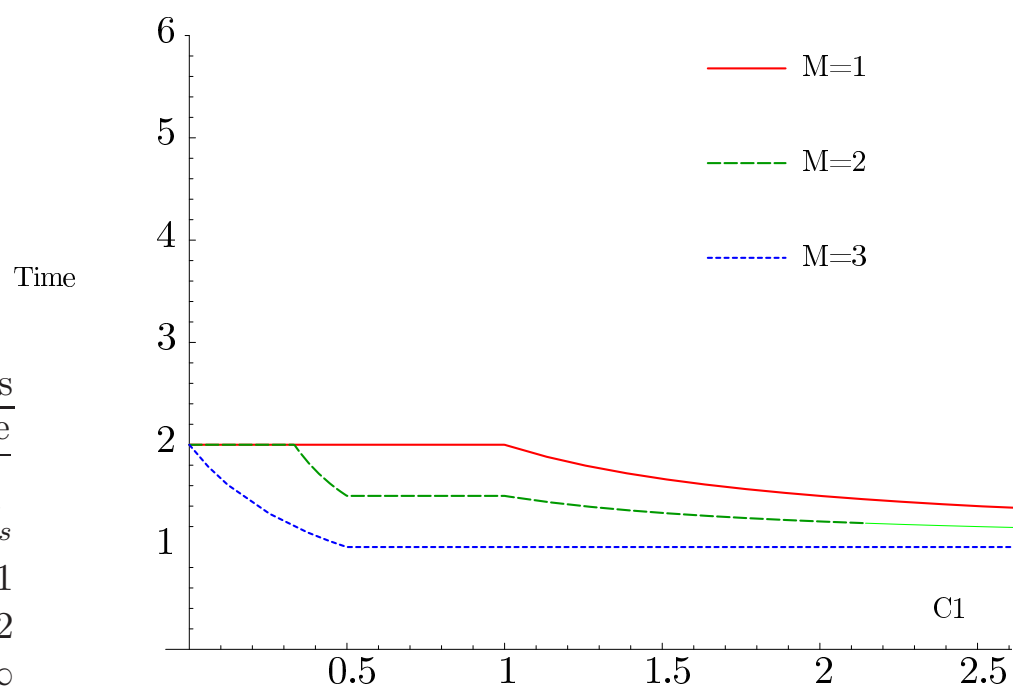

Figure 2: Minimal makespan as a function of $C_{1} / C_{S}$ for different values of $M$ when $N=2$.

In the next section, we extend the results in this limiting case to a much more general scenario. 


\section{Centralized Fluid Limit Solution}

In this section, we generalize the results of Section 4.4 to allow for general capacities $C_{i}$. Moreover, instead of limiting the number of sources to one designated server with a file to disseminate, we now allow every user $i$ to have a file that is to be disseminated to all other users. We provide the centralized solution in the limiting case that the file can be divided into infinitely many parts.

Let $F_{i} \geq 0$ denote the size of the file that user $i$ disseminates to all other users. Seeing that in this situation there is no longer one particular server and everything is symmetric, we change notation for the rest of this section so that there are $N \geq 2$ users $1,2, \ldots, N$. Moreover, let $F=\sum_{i=1}^{N} F_{i}$ and $C=\sum_{i=1}^{N} C_{i}$. We will prove the following result.

Theorem 4 In the fluid limit, the minimal makespan is

$$
T^{*}=\max \left\{\frac{F_{1}}{C_{1}}, \frac{F_{2}}{C_{2}}, \ldots, \frac{F_{N}}{C_{N}}, \frac{(N-1) F}{C}\right\}
$$

and this can be achieved with a two-hop strategy, i.e., one in which users i's file is uploaded to user $j$, either directly from user $i$, or via at most one intermediate user.

Proof. The result is obvious for $N=2$. Then the minimal makespan is $\max \left\{F_{1} / C_{1}, F_{2} / C_{2}\right\}$ and this is exactly the value of $T^{*}$ in (21).

So we consider $N \geq 3$. It is easy to see that each of the $N+1$ terms within the braces on the right hand side of (21) are lower bounds on the makespan. Each user has to upload his file at least to one user, which takes time $F_{i} / C_{i}$. Moreover, the total volume of files to be uploaded is $(N-1) F$ and the total available capacity is $C$. Thus, the makespan is at least $T^{*}$, and it remains to be shown that a makespan of $T^{*}$ can be achieved. There are two cases to consider.

Case 1: $(N-1) F / C \geq \max _{i} F_{i} / C_{i}$ for all $i$.

In this case, $T^{*}=(N-1) F / C$. Let us consider the 2-hop strategy in which each user uploads a fraction $\alpha_{i i}$ of its file $F_{i}$ directly to all $(N-1)$ peers, simultaneously and at equal rates. Moreover, he uploads a fraction $\alpha_{i j}$ to peer $j$ who in turn then uploads it to the remaining $(N-2)$ peers, again simultaneously and at equal rates. Note that $\sum_{j=1}^{N} \alpha_{i j}=1$.

Explicitly constructing a suitable set $\alpha_{i j}$, we thus obtain the problem

$$
\min T
$$

subject to, for all $i$,

$$
\frac{1}{C_{i}}\left[\alpha_{i i} F_{i}(N-1)+\sum_{k \neq i} \alpha_{i k} F_{i}+\sum_{k \neq i} \alpha_{k i} F_{k}(N-2)\right] \leq T .
$$

We minimize $T$ by choosing the $\alpha_{i j}$ in such a way as to equate the $N$ left hand sides of the constraints, if possible. Rewriting the expression in square brackets, equating the constraints 
for $i$ and $j$ and then summing over all $j$ we obtain

$$
\begin{aligned}
& C\left[\alpha_{i i} F_{i}(N-2)+F_{i}+\sum_{k \neq i} \alpha_{k i} F_{k}(N-2)\right] \\
& =C_{i}\left[(N-2) \sum_{j} \alpha_{j j} F_{j}+F+(N-2)\left(F-\sum_{j} \alpha_{j j} F_{j}\right)\right] \\
& =(N-1) C_{i} F .
\end{aligned}
$$

Thus,

$$
\alpha_{i i} F_{i}(N-2)+F_{i}+\sum_{k \neq i} \alpha_{k i} F_{k}(N-2)=(N-1) \frac{C_{i}}{C} F .
$$

Note that there is a lot of freedom in the choice of the $\alpha$ so let us specify that we require $\alpha_{k i}$ to be constant in $k$ for $k \neq i$, that is $\alpha_{k i}=\alpha_{i}^{*}$ for $k \neq i$. This means that $i$ has the capacity to take over a certain part of the dissemination from some peer, then it can and will also take over the same proportion from any other peer. Put another way, user $i$ splits excess capacity equally between its peers. Thus,

$$
\alpha_{i i} F_{i}(N-2)+F_{i}+\alpha_{i}^{*}(N-2)\left(F-F_{i}\right)=(N-1) \frac{C_{i}}{C} F
$$

Still, we have twice as many variables as constraints. Let us also specify that $\alpha_{i}^{*}=\alpha_{i i}$ for all $i$. Similarly as above, this says that the proportion of its own file $F_{i}$ that $i$ uploads to all its peers (rather than just to one of them) is the same as the proportion of the files that it takes over from its peers. Then

$$
\alpha_{i}^{*}=\frac{(N-1)\left(C_{i} / C\right) F-F_{i}}{(N-2) F}=\frac{(N-1) C_{i}}{(N-2) C}-\frac{F_{i}}{(N-2) F}
$$

where $\sum_{i} \alpha_{i}^{*}=1$ and $\alpha_{i}^{*} \geq 0$, because in case $1 F_{i} / C_{i} \leq(N-1) F / C$.

With these $\alpha_{i j}$, we obtain the time for $i$ to complete its upload and hence the time for everyone to complete their upload as

$$
\begin{aligned}
T & =\frac{1}{C_{i}}\left[\alpha_{i}^{*} F_{i}(N-2)+F_{i}+\sum_{k \neq i} \alpha_{i}^{*} F_{k}(N-2)\right] \\
& =\frac{(N-1) F_{i}}{C}-\frac{F_{i}{ }^{2}}{C_{i} F}+\frac{F_{i}}{C_{i}}+\frac{(N-1)\left(F-F_{i}\right)}{C}-\frac{F_{i}\left(F-F_{i}\right)}{C_{i} F} \\
& =(N-1) F / C .
\end{aligned}
$$

Note that there is no problem with precedence constraints. All uploads happen simultaneously stretched out from time 0 to $T$. User $i$ uploads to $j$ a fraction $\alpha_{i j}$ of $F_{i}$. Thus, he does so at constant rate $\alpha_{i j} F_{i} / T_{i}=\alpha_{i j} F_{i} / T$. User $j$ passes on the same amount of data to each of the other users in the same time, hence at the same rate $\alpha_{i j} F_{i} / T_{j}=\alpha_{i j} F_{i} / T$.

Thus, we have shown that if the aggregate lower bound dominates the others, it can be achieved. It remains to be shown that if an individual lower bound dominates, than this can be achieved also. 
Case 2: $F_{i} / C_{i}>(N-1) F / C$ for some $i$.

By contradiction it is easily seen that this cannot be the case for all $i$. Let us order the users in decreasing order of $F_{i} / C_{i}$, so that $F_{1} / C_{1}$ is the largest of the $F_{i} / C_{i}$. We wish to show that all files can be disseminated within a time of $F_{1} / C_{1}$. To do this we construct new capacities $C_{i}^{\prime}$ with the following properties:

$$
\begin{gathered}
C_{1}^{\prime}=C_{1}, \\
C_{i}^{\prime} \leq C_{i} \text { for } i \neq 1, \\
(N-1) F / C^{\prime}=F_{1} / C_{1}^{\prime}=F_{1} / C_{1} \text { and } \\
F_{i} / C_{i}^{\prime} \leq F_{1} / C_{1} .
\end{gathered}
$$

This new problem satisfies the condition of Case 1 and so the minimal makespan is $T^{\prime}=$ $F_{1} / C_{1}$. Hence the minimal makespan in the original problem is $T=F_{1} / C_{1}$ also, because the unprimed capacities are greater or equal to the primed capacities by property (30).

To explicitly construct capacities satisfying (29)-(32), let us define

$$
C_{i}^{\prime}=(N-1) \frac{C_{1}}{F_{1}} \gamma_{i} F_{i}
$$

with constants $\gamma_{i} \geq 0$ such that

$$
\sum_{i} \gamma_{i} F_{i}=F
$$

Then $(N-1) F / C^{\prime}=F_{1} / C_{1}$, that is (31) holds. Moreover, choosing

$$
\gamma_{i} \leq \frac{1}{N-1} \frac{C_{i}}{F_{i}} \frac{F_{1}}{C_{1}}
$$

ensures $C_{i}^{\prime} \leq C_{i}$, i.e. property (30) and choosing

$$
\gamma_{i} \geq \frac{1}{N-1}
$$

ensures $F_{i} / C^{\prime} i \leq F_{1} / C_{1}$, that is property (32). Furthermore, the previous two conditions together ensure that $\gamma_{1}=1 /(N-1)$ and thus $C_{1}^{\prime}=C_{1}$, that is property (29). It remains to construct a set of parameters $\gamma_{i}$ that satisfies (34), (35) and (36).

Putting all $\gamma_{i}$ equal to the lower bound (36) gives $\sum_{i} \gamma_{i} F_{i}=F /(N-1)$, that is too small to satisfy (34). Putting all equal to the upper bound (35) gives $\sum_{i} \gamma_{i} F_{i}=F_{1} C /(N-1) C_{1}$, that is too large to satisfy (34). So we pick a suitably weighted average instead. Namely,

$$
\gamma_{i}=\frac{1}{N-1}\left[\delta \frac{C_{i}}{F_{i}} \frac{F_{1}}{C_{1}}+(1-\delta)\right]
$$

such that

$$
\delta \frac{C}{N-1} \frac{F_{1}}{C_{1}}+(1-\delta) \frac{F}{N-1}=F
$$

that is

$$
\delta=\frac{(N-2) F C_{1}}{F_{1} C-F C_{1}}
$$


Substituting back in we obtain

$$
\gamma_{i}=\frac{1}{N-1} \frac{(N-2) F F_{1} C_{i}+F_{i} F_{1} C-(N-1) F F_{i} C_{1}}{\left(F_{1} C-F C_{1}\right) F_{i}}
$$

and thus

$$
C_{i}^{\prime}=\frac{C_{1}}{F_{1}} \frac{(N-2) F F_{1} C_{i}+F_{i} F_{1} C-(N-1) F F_{i} C_{1}}{F_{1} C-F C_{1}}
$$

By construction, these $C_{i}^{\prime}$ satisfy properties (29)-(32) and hence, by the results in Case $1, T^{\prime}=F_{1} / C_{1}$. Hence the minimal makespan in the original problem $T=F_{1} / C_{1}$ also.

It is worth noting that there is a lot of freedom in the choice of the $\alpha_{i j}$. We have chosen a symmetric approach, but other choices are possible.

In practice, the file will not be infinitely divisible. However, we often have $M>>\log (N)$ and this appears to be sufficient for (21) to be a good approximation. Thus, the fluid limit approach of this section is suitable for typical and for large values of $M$.

\section{Decentralized Solution for Equal Capacities}

In order to give a lower bound on the minimal makespan, we have been assuming a centralized controller does the scheduling. We now consider a naive randomized strategy and investigate the loss in performance that is due to the lack of centralized control. We do this for equal capacities and in two different information scenarios, evaluating its performance by analytic bounds, simulation as well as direct computation. In Section 6.1 we consider the special case of one file part, in Section 6.2 we consider the general case of $M$ file parts. We find that even this naive strategy disseminates the file in an expected time whose growth rate with $N$ is similar to the growth rate of the minimal time that we have found for a centralized controller (cf. Section 31). This suggests that the performance of necessarily decentralized P2P file dissemination systems should still be close to our performance bounds so that they are useful in practice.

\subsection{The special case of one file part}

\section{Assumptions}

Let us start with the case $M=1$. We must first specify what information is available to users. It makes sense to assume that each peer knows the number of parts into which the file is divided, $M$, and the address of the server. However, a peer might not know $N$, the total number of peers, nor its peers' addresses, nor if they have the file, nor whether they are at present occupied uploading to someone else.

We consider two different information scenarios. In the first one, List, the number of peers holding the file and their addresses are known. In the second one, NoList, the number and addresses of all peers are known, but not which of them currently hold the file. Thus, in List, downloading users choose uniformly at random between the server and the peers already having the file. In NoList, downloading users choose uniformly amongst the server and all their peers. If a peer receives a query from a single peer, he uploads the file to that peer. If a peer receives queries from multiple peers, he chooses one of them uniformly at random. The others remain unsuccessful in that round. Thus, in List transmission can fail only if too 
many users try to download simultaneously from the same uploader. In NoList, transmission might also fail if a user tries to download from a peer who does not yet have the file.

\section{Theoretical Bounds}

The following theorem explains how the expected makespan that is achieved by the randomized strategy grows with $N$, in both the List and the NoList scenarios.

Theorem 5 In the uplink-sharing model, with equal upload capacities, the expected number of rounds required to disseminate a single file to all peers in either the List or NoList scenario is $\Theta(\log N)$.

Proof. In the List scenario our simple randomized algorithm runs in less time than in the NoList scenario. Since already have the lower bound given by Theorem 1 it suffices to prove that the expected runing time in the NoList scenario is $O(\log N)$. There is also similar direct proof that the expected running time under the List scenario is $O(\log N)$.

Suppose we have reached a stage in the dissemination at which $n_{1}$ peers (including the server) have the file and $n_{0}$ peers do not, with $n_{0}+n_{1}=N+1$. (The base case is $n_{1}=1$, when only the server has the file.) Each of the peers that does not have the file randomly chooses amongst the server and all his peers (NoList) and tries to download the file. If more than one peer tries to download from the same place then only one of the downloads is successful. The proof has two steps.

(i) Suppose that $n_{1} \leq n_{0}$. Let $i$ be the server or a peer who has the file and let $I_{i}$ be an indicator random variable that is 0 or 1 as $i$ does or does not upload it. Let $Y=\sum_{i} I_{i}$, where the sum is taken over all $n_{1}$ peers who have the file. Thus $n_{1}-Y$ is the number of uploads that take place. Then

$$
E I_{i}=\left(1-\frac{1}{N}\right)^{n_{0}} \leq\left(1-\frac{1}{2 n_{0}}\right)^{n_{0}} \leq \frac{1}{\sqrt{e}}
$$

Now since $E\left(\sum_{i} I_{i}\right)=\sum_{i} E I_{i}$, we have $E Y \leq n_{1} / \sqrt{e}$. Thus, by the Markov inequality, that for a nonnegative random variable $Y$ we have that for any $k$ (not necessarily an integer) $P(Y \geq k) \leq(1 / k) E Y$, we have by taking $k=(2 / 3) n_{1}$,

$$
P\left(n_{1}-Y \equiv \text { number of uploads } \leq \frac{1}{3} n_{1}\right)=P\left(Y \geq \frac{2}{3} n_{1}\right) \leq \frac{n_{1} / \sqrt{e}}{\frac{2}{3} n_{1}}=3 /(2 \sqrt{e})<1 .
$$

Thus the expected number of steps required for the number of peers who have the file to increases from $n_{1}$ to at least $n_{1}+(1 / 3) n_{1}=(4 / 3) n_{1}$ is bounded by a geometric random variable with mean $\mu=1 /(1-3 /(2 \sqrt{e}))$. This implies that we will reach a state in which more peers have the file than do not in an expected time that is $O(\log N)$. From that point we continue with step (ii) of the proof.

(ii) Suppose $n_{1}>n_{0}$. Let $j$ be a peer who does not have the file and let $J_{j}$ be an indicator random variable that is 0 or 1 as peer $j$ does or does not succeed in downloading it. Let $Z=\sum_{j} J_{j}$, where the sum is taken over all $n_{0}$ peers who do not have the file. Suppose $X$ is the number of the other $n_{0}-1$ peers that try to download from the same place as does peer 
$j$. Then

$$
\begin{aligned}
P\left(J_{j}=0\right) & =E\left[\frac{n_{1}}{N}\left(\frac{1}{1+X}\right)\right] \\
& \geq E\left[\frac{n_{1}}{N}(1-X)\right] \\
& =\frac{n_{1}}{N}\left(1-\frac{n_{0}-1}{N}\right) \\
& =\frac{n_{1}}{N}\left(1-\frac{N-n_{1}}{N}\right) \\
& =\frac{n_{1}^{2}}{N^{2}} \\
& \geq 1 / 4 .
\end{aligned}
$$

Hence $E Z \leq(3 / 4) n_{0}$ and so, again using the Markov inequality,

$$
P\left(n_{0}-Z \equiv \text { number of downloads } \leq \frac{1}{8} n_{0}\right)=P\left(Z \geq \frac{7}{8} n_{0}\right) \leq \frac{\frac{3}{4} n_{0}}{\frac{7}{8} n_{0}}=\frac{6}{7} .
$$

It follows that the number of peers who do not yet have the file decreases from $n_{0}$ to no more than $(7 / 8) n_{0}$ in an expected number of steps no more than $\mu^{\prime}=1 /\left(1-\frac{6}{7}\right)=7$. Thus the number of steps needed for the number of peers without the file to decrease from $n_{0}$ to 0 is $O\left(\log n_{0}\right)=O(\log N)$. In fact, this is a weak upper bound. By more complicated arguments we can show that if $n_{0}=a N$, where $a \leq 1 / 2$, then the expected remaining time for our algorithm to complete under NoList is $\Theta(\log \log N)$. For $a>1 / 2$ the expected time remains $\Theta(\log N)$.

\section{Simulation}

For the problem with one server and $N$ users we have carried out 1000 independent simulation runs $^{4}$ for a large range of parameters, $N=2,4, \ldots, 2^{25}$. We found that the achieved expected makespan appears to grow as $a+b \times \log _{2} N$. Motivated by this and the theoretical bound from Theorem 5 we fitted the linear model

$$
y_{i j}=\alpha+\beta x_{i}+\epsilon_{i j}
$$

where $y_{i j}$ is the makespan for $x_{i}=\log _{2} 2^{i}$, obtained in run $j, j=1, \ldots, 1000$. Indeed, the model fits the data very well in both scenarios. We obtain the following results that enable us to compare the expected makespan of the naive randomized strategy to the that of a centralized controller.

For List, the regression analysis gives a good fit, with Multiple R-squared value of 0.9975 and significant p- and t-values. The makespan increases as

$$
1.1392+1.1021 \times \log _{2} N .
$$

\footnotetext{
${ }^{4}$ As many as 1000 runs were required for the comparison with the computational results in Tables 4 and 5 mainly because the makespan always takes integer values.
} 
For NoList, there is more variation in the data than for List, but, again, the linear regression gives a good fit, with Multiple R-squared of 0.9864 and significant p- and t-values. The makespan increases as

$$
1.7561+1.5755 \times \log _{2} N .
$$

As expected, the additional information for List leads to a significantly lesser makespan when compared to NoList, in particular the log-term coefficient is significantly smaller. In the List scenario, the randomized strategy achieves a makespan that is very close to the centralized optimum of $1+\left\lfloor\log _{2} N\right\rfloor$ of Section B It is only suboptimal by about $10 \%$. Hence even this simple randomized strategy performs well in both cases and very well when state information is available, suggesting that our bounds are useful in practice.

\section{Computations}

Alternatively, it is possible to compute the mean makespan analytically by considering a Markov Chain on the state space $0,1,2, \ldots, N$, where state $i$ corresponds to $i$ of the $N$ peers having the file. We can calculate the transition probabilities $p_{i j}$. In the NoList case, for example, following the Occupancy Distribution (e.g., [18]), we obtain

$$
p_{i i+m}=\sum_{j=i-m}^{i} \frac{(-1)^{j-i+m} i !}{(i-j) !(i-m) !(j-i+m) !}\left(\frac{N-1-j}{N-1}\right)^{N-i} .
$$

Hence we can successively compute the expected hitting times $k(i)$ of state $N$ starting from state $i$ via

$$
k(i)=\frac{1+\sum_{j>i} k(j) p_{i j}}{1-p_{i i}} .
$$

The resulting formula is rather complicated, but can be evaluated exactly using arbitrary precision arithmetic on a computer. Computation times are long, so to keep them shorter we only work out the transition probabilities of the associated Markov Chain exactly. Hitting times are then computed in double arithmetic, that is, to 16 significant digits. Even so, computations are only feasible up to $N=512$ with our equipment, despite repeatedly enhanced efficiency. This suggests that simulation is the more computationally efficient approach to our problem. The computed mean values for List and NoList are shown in Tables 4 and 5 respectively. The difference to the simulated values is small without any apparent trend. It can also be checked by computing the standard deviation that the computed mean makespan is contained in the approximate $95 \%$ confidence interval of the simulated mean makespan. The only exception is for $N=128$ for NoList where it is just outside by approximately 0.0016 .

Thus, the computations prove our simulation results accurate. Since simulation results are also obtained more efficiently, we shall stick to simulation when investigating the general case of $M$ file parts in the next section.

\subsection{The general case of $M$ file parts}

\section{Assumptions}

We now consider splitting the file into several file parts. With the same assumptions as in the previous section, we repeat the analysis for List for various values of $M$. Thus, in each round, a downloading user connects to a peer chosen uniformly at random from those peers 


\begin{tabular}{rrrr}
\hline$N$ & sim. & comp. & difference \\
\hline 2 & 2.000 & 2.000 & $=0.000$ \\
4 & 3.089 & 3.083 & +0.006 \\
8 & 4.167 & 4.172 & -0.005 \\
16 & 5.333 & 5.319 & +0.014 \\
32 & 6.534 & 6.538 & -0.004 \\
64 & 7.806 & 7.794 & +0.012 \\
128 & 8.994 & 8.981 & +0.013 \\
256 & 10.059 & 10.057 & +0.002 \\
512 & 11.107 & 11.116 & -0.009 \\
\hline
\end{tabular}

Table 4: Simulated and computed mean makespans for List are close.

\begin{tabular}{rrrr}
\hline$N$ & sim. & comp. & difference \\
\hline 2 & 2.314 & 2.333 & -0.019 \\
4 & 4.071 & 4.058 & +0.013 \\
8 & 5.933 & 5.956 & -0.023 \\
16 & 7.847 & 7.867 & -0.020 \\
32 & 9.689 & 9.710 & -0.021 \\
64 & 11.430 & 11.475 & -0.045 \\
128 & 13.092 & 13.173 & -0.081 \\
256 & 14.827 & 14.819 & +0.008 \\
512 & 16.426 & 16.427 & -0.001 \\
\hline
\end{tabular}

Table 5: Simulated and computed mean makespans for NoList are close.

that have at least one file part that the user does not yet have. An uploading peer randomly chooses one out of the peers requesting a download from him. He uploads to that peer a file part that is randomly chosen from amongst those that he has and the peer still needs.

\section{Simulation}

Again, we consider a large range of parameter. We carried out 100 independent runs for each $N=2,4, \ldots, 2^{15}$. For each value of $M=1-5,8,10,15,20,50$ we fitted the linear model (46).

Table 6 summarizes the simulation results. The Multiple R-squared values indicate a good fit, although the fact that these decrease with $M$ suggests there may be a finer dependence on $M$ or $N$. In fact, we obtain a better fit using Generalized Additive Models (cf. 14]). However, our interest here is not in fitting the best possible model, but to compare the growth rate with $N$ to the one obtained in the centralized case in Section 3. Moreover, from the diagnostic plots we note that the actual performance for large $N$ is better than given by the regression line, increasingly so for increasing $M$. In each case, we obtain significant p- and t-values. The regression $0.7856+1.1520 \times \log _{2} N$ for $M=1$ does not quite agree with $1.1392+1.1021 \times \log _{2} N$ found in (47). It can be checked, by repeating the analysis there for $N=2,4, \ldots, 2^{15}$ that this is due to the different range of $N$. Thus, our earlier result of 1.1021 might be regarded more reliable, being based on $N$ ranging up to $2^{25}$.

We conclude that, as in the centralized scenario, the makespan can also be reduced significantly in a decentralized scenario even when a simple randomized strategy is used to disseminate the file parts. However, as we note by comparing the second and fourth columns of Table [6] as $M$ increases the achieved makespan compares less well relative to the centralized minimum of $1+(1 / M)\left\lfloor\log _{2} N\right\rfloor$. In particular, note the slower decrease of the log-term coefficient. This is depicted in Figure 3

Still, we have seen that even this naive randomized strategy disseminates the file in an expected time whose growth rate with $N$ is similar to the growth rate of the minimal time that we have found for a centralized controller in Section 3 , confirming our performance bounds are useful in practice. This is confirmed also by initial results of current work on the performance evaluation of the Bullet' system [20].

The program code for simulations as well as the computations and the diagnostic plots 


\begin{tabular}{rccc}
\hline$M$ & $\begin{array}{c}\text { Fitted } \\
\text { makespan }\end{array}$ & $\begin{array}{c}\text { Multiple } \\
\text { R-squared }\end{array}$ & $1 / M$ \\
\hline 1 & $0.7856+1.1520 \times \log _{2} N$ & 0.9947 & 1.000 \\
2 & $1.3337+0.6342 \times \log _{2} N$ & 0.9847 & 0.500 \\
3 & $1.4492+0.4561 \times \log _{2} N$ & 0.9719 & 0.333 \\
4 & $1.4514+0.3661 \times \log _{2} N$ & 0.9676 & 0.250 \\
5 & $1.4812+0.3045 \times \log _{2} N$ & 0.9690 & 0.200 \\
8 & $1.4907+0.2113 \times \log _{2} N$ & 0.9628 & 0.125 \\
10 & $1.4835+0.1791 \times \log _{2} N$ & 0.9602 & 0.100 \\
15 & $1.4779+0.1326 \times \log _{2} N$ & 0.9530 & 0.067 \\
20 & $1.4889+0.1062 \times \log _{2} N$ & 0.9449 & 0.050 \\
50 & $1.4524+0.0608 \times \log _{2} N$ & 0.8913 & 0.020 \\
\hline
\end{tabular}

Table 6: Simulation results in the decentralized List scenario for various values of $M$ and $\log$-term coefficients in the centralized optimum (cf. Theorem \).

used in this section are available on request and will be made available via the Internet $^{5}$.

\section{Discussion}

In this paper, we have given three complementary solutions for the minimal time to fully disseminate a file of $M$ parts from a server to $N$ end users in a centralized scenario, thereby providing a lower bound on and a performance benchmark for P2P file dissemination systems. Our results illustrate how the $\mathrm{P} 2 \mathrm{P}$ approach, together with splitting the file into $M$ parts, can achieve a significant reduction in makespan. Moreover, the server has a reduced workload when compared to the traditional client/server approach in which it does all the uploads itself. We also investigate the part of the loss in efficiency that is due to the lack of centralized control in practice. This suggests that the performance of necessarily decentralized P2P file dissemination systems should still be close to our performance bound confirming their practical use.

It would now be very interesting to compare dissemination times of the various efficient real overlay networks directly to our performance bound. A mathematical analysis of the protocols is rarely tractable, but simulation or measurements such as in [17] and [30] for the BitTorrent protocol can be carried out in an environment suitable for this comparison. Cf. also testbed results for Slurpie [33] and simulation results for Avalanche [12. It is current work to compare our bounds to the makespan obtained by Bullet' 20. Initial results confirm their practical use further.

In practice, splitting the file and passing on extra information has an overhead cost. Moreover, with the Transmission Control Protocol (TCP), longer connections are more efficient than shorter ones. TCP is used practically everywhere except for the Internet Control Message Protocol (ICMP) and User Datagram Protocol (UDP) for real-time applications. For further details see [35. Still, with an overhead cost it will not be optimal to increase $M$ beyond a certain value. This could be investigated in more detail.

In the proof of Lemma 1 and Lemma 2 we have used fair sharing and continuity assumptions. It would be of interest to investigate whether one of them or both can be relaxed.

\footnotetext{
${ }^{5}$ http://www.statslab.cam.ac.uk/ jm288/
} 


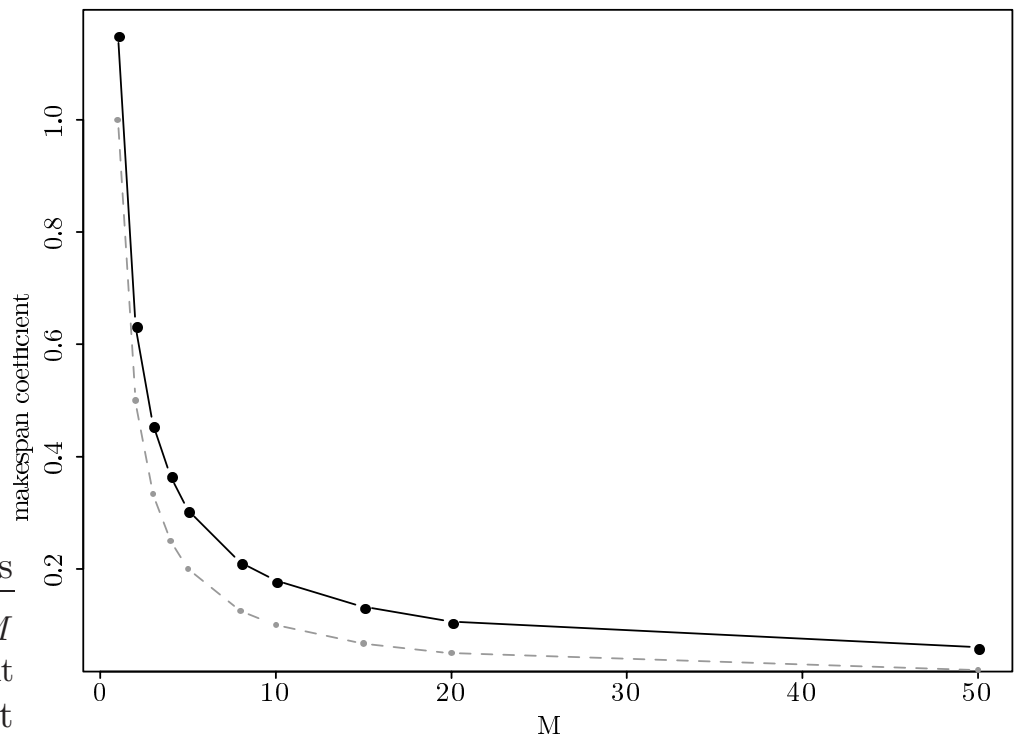

Figure 3: Illustration of the log-term coefficients of the makespan from Table 6] the decentralized List scenario (solid) and the idealized centralized scenario (dashed).

It would be interesting to generalize our results to account for a dynamic setting with peers arriving and perhaps leaving when they have completed the download of the file. In Internet applications users often connect for only relatively short times. Work in this direction, using a fluid model to study the steady-state performance, is pursued in [31] and there is other relevant work in [37.

Also of interest would be to extend our model to consider users who prefer to free-ride and do not wish to contribute uploading effort. Or, to users who might want to leave the system once they have downloaded the whole file, a behaviour sometimes referred to as easy-riding. The BitTorrent protocol, for example, implements a choking algorithm to limit free-riding.

In another scenario it might be appropriate to assume that users push messages rather than pull them. See [1] for an investigation of the design space for distributed information systems. The push-pull distinction is also part of their classification. In a push system, the centralized case would remain the same. However, we expect the decentralized case to be different. There are a number of other interesting questions which could be investigated in this context. For example, what happens if only a subset of the users is actually interested in the file, but the uploaders do not know which.

From a mathematical point of view it would also be interesting to consider additional download constraints explicitly as part of the model, in particular when up- and download capacities are all different and not positively correlated. We might suppose that user $i$ can upload at a rate $C_{i}$ and simultaneously download at rate $D_{i}$.

More generally, one might want to assume different capacities for all links between pairs. Or, phrased in terms of transmission times, let us assume that for a file to be sent from user $i$ to user $j$ it takes time $t_{i j}$. Then we obtain a transportation network, where instead of link costs we now have link delays. This problem can be phrased as a one-to-all shortest path problem if $C_{j}$ is at least $N+1$. This suggests that there might be some relation which could be exploited. On the other hand, the problem is sufficiently different so that greedy algorithms, induction on nodes and Dynamic Programming do not appear to work. Background on these 
can be found in [4] and [3]. For $M=1$, Prüfer's $(N+1)^{N-1}$ labelled trees [6] together with the obvious $O(N)$ algorithm for the optimal scheduling given a tree is an exhaustive search. A Branch and Bound algorithm can be formulated.

\section{References}

[1] R. Ahlswede, N. Cai, S.-Y. R. Li, and R. W. Yeung. Network information flow. IEEE Trans. on Information Theory, 46:1204-1216, 2000.

[2] A. Bar-Noy, S. Kipnis, and B. Schieber. Optimal multiple message broadcasting in telephone-like communication systems. Discrete Applied Mathematics, 100:1-15, 2000.

[3] M. S. Bazaraa, J. J. Jarvis, and H. D. Sharali. Linear Programming and Network Flows. Wiley, 3rd edition, 2005.

[4] D. Bertsimas and J. N. Tsitsiklis. Introduction to Linear Optimization. Athena Scientific, Belmont, 1997.

[5] A. R. Bharambe, C. Herley, and V. N. Padmanabhan. Some observations on BitTorrent performance. Performance Evaluation Review, 33(1):398-399, 2005.

[6] B. Bollobás. Modern Graph Theory. Springer, New York, 1998.

[7] C. Castro, P. Druschel, A.-M. Kermarrec, A. Nandi, A. Rowstron, and A. Singh. SplitStream: High-bandwidth multicast in cooperative environments. In 19th ACM Symposium on Operating Systems Principles (SOSP'03), 2003.

[8] E. J. Cockayne and A. G. Thomason. Optimal multimessage broadcasting in complete graphs. Utilitas Math., 18:181-199, 1980.

[9] B. Cohen. Incentives build robustness in BitTorrent. Proceedings of the Workshop on Economics of Peer-to-Peer Systems, Berkeley, CA, 2003.

[10] A. M. Farley. Broadcast time in communication networks. SIAM Journal on Applied Mathematics, 39(2):385-390, October 1980.

[11] M. Franklin and S. Zdonik. A framework for scalable dissemination-based systems. ACM SIGPLAN Notices, 32(10):94 - 105, 1997.

[12] C. Gkantsidis and P. Rodriguez. Network coding for large scale content distribution. In IEEE INFOCOM 2005, March 2005.

[13] L. Guo, S. Chen, Z. Xiao, E. Tan, X. Ding, and X. Zhang. Measurements, analysis, and modeling of BitTorrent-like systems. In Proceedings of Internet Measurement Conference 2005 (IMC 2005), October 2005.

[14] T. Hastie and R. Tibshirani. Generalized Additive Models. Chapman and Hall, London, 1990.

[15] S. M. Hedetniemi, S. T. Hedetniemi, and A. L. Liestman. A survey of gossiping and broadcasting in communication networks. Networks, 18(4):319-349, 1988. 
[16] J. Hromkovic, R. Klasing, B. Monien, and R. Peine. Combinatorial Network Theory (F. Hsu and D.-Z. Du, Eds.), chapter Dissemination of Information in Interconnection Networks (Broadcasting and Gossiping), pages 125-212. Kluwer Academic Publishers, 1995.

[17] M. Izal, G. Urvoy-Keller, E. Biersack, P. Felber, A. A. Hamra, and L. Garcés-Erice. Dissecting BitTorrent: Five months in a torrent's lifetime. Passive and Active Measurements $2004,2004$.

[18] N. L. Johnson, S. Kotz, and A. W. Kemp. Univariate Discrete Distributions. Wiley Europe, 1993.

[19] T. Karagiannis, A. Broido, N. Brownlee, k. claffy, and M. Faloutsos. Is P2P dying or just hiding? In IEEE Globecom 2004 - Global Internet and Next Generation Networks, November 2004.

[20] D. Kostić, R. Braud, C. Killian, E. Vandekieft, J. W. Anderson, A. C. Snoeren, and A. Vahdat. Maintaining high-bandwidth under dynamic network conditions. In USENIX 2005 Annual Technical Conference, 2005.

[21] C.-H. Kwon and K.-Y. Chwa. Multiple message broadcasting in communication networks. Networks, 26:253-261, 1995.

[22] L. Massoulié and M. Vojnović. Coupon replication systems. In ACM Sigmetrics 2005, June 2005.

[23] J. Mundinger. Analysis of Peer-to-Peer Systems in Communication Networks. PhD thesis, Cambridge University, August 2005.

[24] J. Mundinger and R. R. Weber. Efficient content distribution using peer-to-peer technology. In C. Griwodz, T. P. Plagemann and R. Steinmetz. 04201 Abstracts Collection - Content Distribution Infrastructures. Dagstuhl Seminar Proceedings, 2004.

[25] J. Mundinger and R. R. Weber. Efficient file dissemination using peer-to-peer technology. Technical Report 2004-01, Statistical Laboratory Research Reports, 2004.

[26] J. Mundinger, R. R. Weber, and G. Weiss. Analysis of peer-to-peer file dissemination. To appear in Performance Evalution Review, Eighth Workshop on Mathematical Performance Modeling and Analysis (MAMA 2006) Issue, 2006.

[27] J. Mundinger, R. R. Weber, and G. Weiss. Analysis of peer-to-peer file dissemination amongst users of different upload capacities. To appear in Performance Evaluation Review, Performance 2005 Issue, 2006.

[28] G. L. Nemhauser and L. A. Wolsey. Integer and Combinatorial Optimization. Wiley, New York, 1988.

[29] A. Parker. The true picture of peer-to-peer filesharing. CacheLogic, 2004. http://www.cachelogic.com/

[30] J. A. Pouwelse, P. Garbacki, D. H. J. Epema, and H. J. Sips. The Bittorrent P2P filesharing system: Measurements and analysis. In 4th International Workshop on Peer-toPeer Systems (IPTPS'05), February 2005. 
[31] D. Qiu and R. Srikant. Modeling and performance analysis of BitTorrent-like peer-to-peer networks. In Proc. ACM SIGCOMM, September 2004.

[32] K. K. Ramachandran and B. Sikdar. An analytic framework for modeling peer to peer networks. IEEE INFOCOM 2005, 2005.

[33] R. Sherwood, R. Braud, and B. Bhattacharjee. Slurpie: A cooperative bulk data transfer protocol. IEEE INFOCOM 2004, 2004.

[34] G. Sierksma. Linear and Integer Programming: Theory and Practice, chapter 6.3. Marcel Dekker, Inc., New York, 2002.

[35] R. Srikant. The Mathematics of Internet Congestion Control. Birkhäuser, 2004.

[36] X. Yang and G. de Veciana. Service capacity of peer to peer networks. IEEE INFOCOM $2004,2004$.

[37] X. Yang and G. de Veciana. Performance of peer-to-peer networks: Service capacity and role of resource sharing policies. Performance Evaluation, Special Issue on Performance Modeling and Evaluation of P2P Computing Systems, 2005.

[38] H.-J. Zimmermann. Operations Research. Vieweg, 2004. 